\title{
Fireground location understanding by semantic linking of visual objects and building information models
}

\author{
FLORIAN VANDECASTEELE ${ }^{1}$, BART MERCI ${ }^{2}$ and STEVEN VERSTOCKT ${ }^{1}$ \\ ${ }^{1}$ Ghent University IMEC, ELIS Department - IDlab \\ Sint-Pietersnieuwstraat, 41, 9000 Ghent, Belgium \\ 2 Ghent University - Department of Flow, Heat and Combustion Mechanics \\ Sint-Pietersnieuwstraat 41, 9000 Ghent, Belgium
}

\begin{abstract}
This paper presents an outline for improved localization and situational awareness in fire emergency situations based on semantic technology and computer vision techniques. The novelty of our methodology lies in the semantic linking of video object recognition results from visual and thermal cameras with Building Information Models (BIM). The current limitations and possibilities of certain building information streams in the context of fire safety or fire incident management are addressed in this paper. Furthermore, our data management tools match higher-level semantic metadata descriptors of BIM and deep-learning based visual object recognition and classification networks. Based on these matches, estimations can be generated of camera, objects and event positions in the BIM model, transforming it from a static source of information into a rich, dynamic data provider. Previous work has already investigated the possibilities to link BIM and lowcost point sensors for fireground understanding, but these approaches did not take into account the benefits of video analysis and recent developments in semantics and feature learning research. Finally, the strengths of the proposed approach compared to the state-of-the-art is its (semi-)automatic workflow, generic and modular setup and multi-modal strategy, which allows to automatically create situational awareness, to improve localization and to facilitate the overall fire understanding.
\end{abstract}

KEYWORDS: building information models, fire analysis, multi-view sensing, location estimation, visual object recognition, semantic linking

\section{INTRODUCTION}

On the one hand, when a fire fighter crew arrives at a fire scene the set-up is unknown, although the commander in chief needs to take fast and appropriate decisions. The location of the fire source, the amount and the position of the victims, the structure and the lay-out of the building, and the health and the location of the firefighters are mostly unknown factors that could influence the firefighting strategy. On the other hand, there is more and more geographic data stored and used almost daily in many organizations. However, the real utilization of geo-information, such as building maps and real-time sensing data is still limited in the analysis of fire emergency situations. Furthermore, there is currently no system that helps in understanding and linking the actual state of the building and the fire behavior during a fire.

The goal of this is paper is to focus on the state-of-the-art in building information models, location understanding for fire incident management and situational awareness.Secondly, an outline is provided to combine the different sources of information involved in fireground analysis. For example, by combining the information of the building model with the results of state-of-the-art computer vision techniques applied on video sensors, an improved understanding of the fireground scene can be achieved. Furthermore, object detection, scene classification and room layout estimation will help in localizing fire fighters, which is a difficult task due to the reduced visibility and dynamic characteristics of the fire environment. The information gained by the proposed architecture could even be used to verify or to update the building information models, which increases their practical applicability. Figure 1 gives a global overview of the proposed approach and shows the links between BIM, visual analysis and the localization problem. Finally, it is important to remark that the proposed framework is work in progress and subject to change over time. 


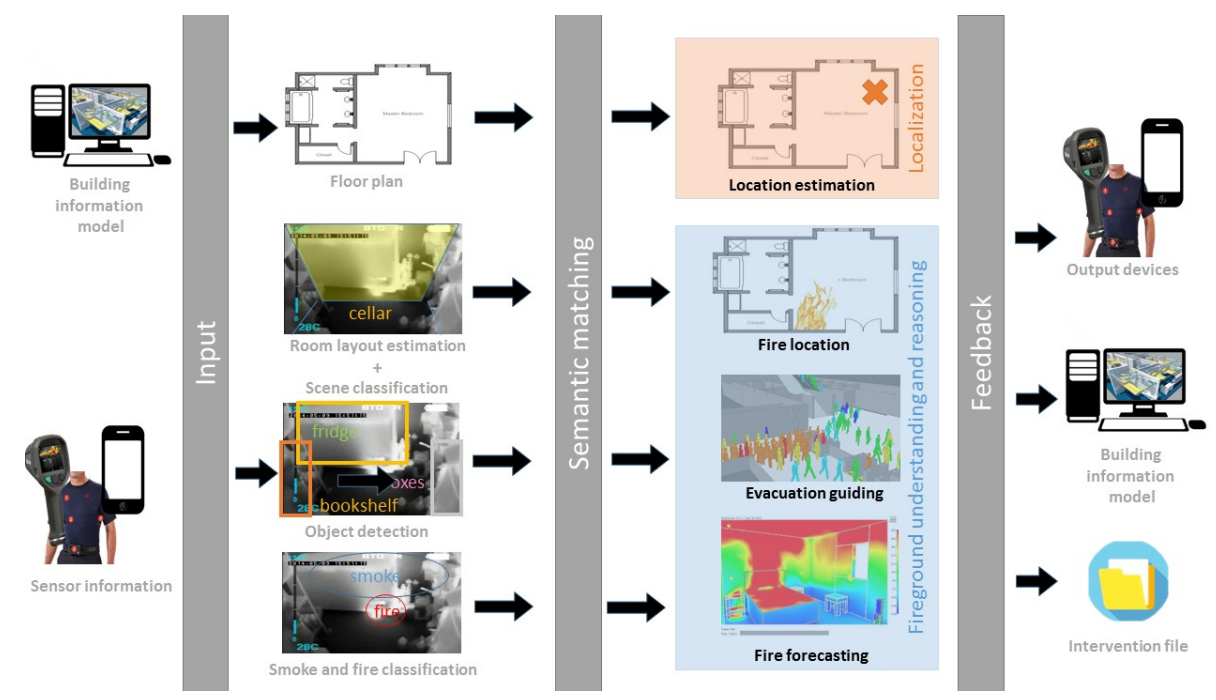

Fig. 1. Global overview of the proposed system for semantic linking of video object recognition results with building information models.

The remainder of this paper is organized as follows. Section 2 presents the global framework for combining low-cost sensing, building information and location understanding. The details of each individual building block are given in the next sections. Section 3 gives a literature overview of building information modeling in the context of fire safety design and fire incident management. Subsequently, Section 4 proposes the possibilities of localization understanding with low-cost video sensors during a fire. Next, Section 5 focuses on the semantic location understanding for location recognition and Section 6 describes the linking process and evaluation methods. Finally, Section 7 lists some conclusions and points out directions for future work.

\section{FIREGROUND UNDERSTANDING AND VISUALIZATION}

The two main goals for combining building information models with real-time sensing data are to improve localization and to facilitate fireground understanding and reasoning. The global framework, shown in Figure 1 , links the following components/modules of both modalities in order to achieve both goals:

- For accurate localization, the floorplan generated from the BIM is combined with the room layout estimation from our low-cost video localization devices. By describing both data types in a semantic way, semantic similarity between both annotations can be used to estimate the location on the floorplan the sensor is focusing on. Furthermore, additional semantic information of our scene classification module can be used to further improve the localization accuracy. However, currently this module only works on visual images. Research is going on to adapt this technique so that is is also applicable on thermal camera images. Both modules improve the situational awareness/localization and facilitate evacuation guiding.

- Semantic annotations of the object detection module and our smoke/fire classification algorithms, on their turn, will continuously update the BIM with real-time information of the fireground. In this way, a detailed spatio-temporal description of the fire evolution can be generated facilitating the overall fire understanding. In combination with the room layout estimation and the scene classification, the object detection module can even be used to perform BIM updates.

- Real-time localized sensing data of the environment (i.e., pressure, smoke layer interface height, smoke and fire location) can be coupled to a fire forecasting mechanism. In combination with BIM, the forecasting mechanism could update the evacuation guiding and generate risk warnings for firefighters. 
- Finally, our detection results are sent back as feedback to our sensing devices and the BIM model. Furthermore, a dynamic, self-updating intervention file could be generated with our real-time detection results. This logging data can, for example, be useful in post-intervention analysis.

Although the building information model is a rich source of information it is important to remark that currently, not many buildings have a decent model. This limits its applicability in the proposed architecture. However, by showing the possibilities of BIM in systems like ours, we believe that its availability and accuracy will increase, making it a rich source of information for a wide variety of applications.

Besides the technical issues, which are the focus of this paper, it is also important to address the factors that will influence the efficiency and the effectivity of the proposed application. Sarshar et al. [1] give some valuable remarks on the usability of smartphone apps in emergencies. In addition, visualization aspects needs also to be evaluated and investigated in our context. There is a large amount of sensing data, building data, and forecasting results that need to be structured and communicated in the final application. User experience studies with the different end users/stakeholders are necessary to adapt the framework's output to their specific needs. The work of Nunavath et al. [2], which analyzes emergency communication during interventions, is already a preliminary step into this direction and can be used as a starting base, but further user experience research is needed. Furthermore, the work of Vandecasteele et al. [3] indicated that a decent visualization can give more insight in the large amount of sensing data during real fire experiments. Recently we also launched a national questionnaire survey for firefighter representatives and leaders focusing on data needs and visualization during an intervention. The discussion of the results and gained insight are out out of scope of this technology-focused research paper, but will be part of our future publications.

Within the next sections, we give a thorough explanation of each of the building blocks of our framework. The most important and novel step, i.e. the semantic matching of the sensor data with the building model, is discussed in Section 6 and performs reasoning on each of the other building blocks' output.

\section{BIM: BUILDING INFORMATION MODEL}

BIM is a tool to manage accurate building information over its whole life cycle [4]. BIM represents the building as models made of collections of building components such as walls, windows, doors and their relationship and properties. Initially it was only intended to support the design and construction processes, but currently there are numerous applications in different fields (e.g. facility management, fire safety engineering, climate control). Furthermore, due to the international Industry Foundation Class (IFC) standard it is now possible to share and store information of other software vendors and to transform the BIM data into semantic understandable data such as XML and RDF-format. In addition to using the building model for incident management it is also suitable to assist decision makers during the fire safety design process (as further discussed in Section 3.1). Furthermore, the building model can also be used for real-time sensing visualization and reasoning (which is described in Section 3.4).

However, like already mentioned before, there are many existing buildings with incomplete or fragmented BIM data, which poses different challenges and problems for its applications. Especially in the firefighting case it is necessary to have up-to-date information of the building/room layout/structure. In order to cope with this issue, Section 5 presents some building blocks (i.e., computer vision modules) that can update the actual state of a room in the BIM model with visual analysis techniques. 


\subsection{Building information for fire safety design}

Decent incident management starts with a good preparation of the fire safety design (and this already in the early design process). This is already extensively discussed in literature. Zou et al.[5], for example, give an overview of the recent trends and possibilities for risk management during the building process. Jelenewicz et al. [6], on the other hand, discuss the use of BIM technology for fire protection in general. Wang et al. [7] present a solution to automate the evacuation route planning.Furthermore, Zhang et al. [8] integrated semantic Natural Language Processing (NLP) for fully-automated building design checking. Evaluating the local or regional regulation codes is an important issue in fire safety design. In the work of Zhang, the BIM information is transformed into logic facts and the semantic reasoner is finally used to verify them against the textual codes. The main advantage of this approach is that the fire safety rules could be checked automatically without manual intervention. A major limitation, however, is that no specific validation is done for fire safety rules, which is our suggestion for their future work. Finally, it's important to remark that a decent fire safety design starts with a representative fire model, the building models could assist in this decision making process.

\subsection{Building models for fire investigation}

Research on building models for fire modeling are in their early phase. Still, fire investigation can be decently used for fire safety design (as mentioned before), or for post-fire analysis. Although, the possible interactions, the building model is currently mostly used for visualization purposes. Wu et al. [9] proposed a system for data interchange between BIM and CFAST (Consolidated Model of Fire and Smoke Transport). After the simulation, simulation results are brought back into the BIM module for visualization. The most interesting block in their approach is the extraction of room configurations and compartment openings from building models. Furthermore, the forecasting with a fast zone-model and the visualization techniques are useful in our context. The disadvantage of their architecture, however, is that there is no verification of the current room configuration and they do not provide updates of the actual state of the building. The building model could be outdated and this could result in inaccurate simulation results. Furthermore, the fire source and location are also not included in their work. As such, their module is only useful for prevention purposes. By incorporating the actual, real-time sensing data, as we propose to do in our approach, the system could be used for situational aware evacuation guiding and localization during a real fire. Lin et al. [10] on the other hand, used a 3D BIM-model for fire cause investigation and visualization. Their work only focuses on presenting different kinds of data for fire disaster analysis and no combination/interaction is made between the sensor data and the building model. As such, the added value of this work is limited in our context.

Besides the data visualization it is necessary to have up-to-date building information for the fire modeling. Franz et al. [11] used the point cloud information of a Google Tango mobile device to generate semiautomated geometric BIM models for post-fire investigation. The major difference is that our data will be used for situational awareness during real fires, which seems to be impossible in their set-up due to the computational complex point-cloud based BIM generation. Experiments have shown that it is not possible to create a decent point cloud model with a Tango device when visibility decreases during a fire. However, the work of Lu et al. [12] showed that it is possible to get image-based localization with thermal images in reduced visibility settings. Within Section 5, a more deeper explanation will be given on the transfer learning aspect for thermal and visual images. Furthermore, the approach of Wills et al. [13] is most closely related to ours. They map sensor data in a three-dimensional BIM for critical fire safety decision making. The most useful/inspirational part of their methodology is the visualization of sensor data of real-fires in a 3D building model and the use of the sensing data for inverse fire model validation. The disadvantage of this approach, however, is that again there is no verification of the actual state of the building model and there is no possibility to integrate dynamic handheld sensors. 
Finally, within the building model there are different parameters available that could assist the fire modeling. Spearpoint et al. [14] investigated the use and the interchangeability of IFC2x2 properties as a source of input data for zone modeling, ranging from building spaces and compartments, over fire suppression and detection systems to material properties (i.e. fire resistance, heat capacity or thermal conductivity). It is important to mention that these values could be used for fire investigation, but also for incident management or evacuation planning. Currently, the IFC4 ${ }^{1}$ is the new standard and compared to the older standard more consistency, connectivity between elements and incorporation of GIS elements are ensured.

\subsection{Building information for evacuation planning}

Besides the use of BIM in fire analysis and forecasting there is also research going on to use BIM to support evacuation planning. Wang et al. [7], for example, used the 3D geometric BIM data and visualization results to support evacuation assessments and escape route planning. Currently, the objects in the building (e.g, furniture and fire safety equipment) are manually registered. With the computer vision based object recognition modules in our framework, an automated understanding and registration of the objects could be made.

Chen et al. [15] use the BIM information to create an evacuation network graph and Zlatanova et al.[16] develop a system for 3D model based emergency evacuation. The output of both systems could be used in our framework for evacuation guiding and routing decisions for rescue operations. The limitations of both systems is that there is also no possibility to integrate sensing data during a fire. Finally, Teo et al. [17] use the geospatial data from BIM and geographic information systems (GIS) for route planning in emergency evacuations. The useful part for our framework is the generation of an indoor network from the BIM/IFC. This could be used for route planning like proposed in the paper, but can also be used for location estimation by applying a coarse-to-fine search approach.

\subsection{Building information combined with real-time sensing}

As indicated before (in Section 2) it is important to have a decent visualization of the real-time sensor data. Wang et al. [18] proposed a conceptual web information service to combine the 3D model and the live sensing data. Their main idea is to use the interface for energy management, but the same mechanism can be used to visualize the sensor data of the temperature [19], the humidity [20] or the CO-level during a fire incident. Finally, the increased use of internet of things (IoT) devices will automate the practical use of real aggregated data. IBM Whatson IoT and Honeywell ${ }^{2}$, for example, already show this combination for reducing energy costs.

\subsection{Future applications of building models}

Beside the usability for evacuation and fire analysis, the information retrieved by the BIM module could also give information to assess further development of the fire. For instance, the main material used for construction (wood, concrete, steal) will result in a different fire behavior and this could be deducted trough the BIM module. The dimensions of the different rooms could be used to calculate the necessary water capacity to extinguish the fire. The level of isolation could indicate if there is a higher risk of pyrolysis and flashover. The level of prevention services (compartments, sprinkler system, smoke extraction systems) can also be indicated in the module and facilitate the commander to make the appropriate decisions. Currently there is no module available that facilitates the communication/visualization of this information. Future research will perform user based evaluations to see the best and easiest way to perform this task.

Another point to address is the BIM data reduction. Currently, the building models have a large amount of data and correlations, which complicates transfer and interpretation. It is not clear if the complete data

\footnotetext{
${ }^{1}$ http://www.buildingsmart-tech.org/specifications/ifc-releases/ifc4-release/buildingSMART_IFC4_Whatisnew.pdf

${ }^{2}$ http://www.ibm.com/internet-of-things/iot-solutions/retail/building-sense/
} 
interchange is always necessary. It could be possible to create a scalable BIM model, which can deliver the specific BIM data that is needed by the application.

An overview of future applications and their links with BIM (as discussed in this section) are shown in Figure 2. The figure shows the commander who receives accurate fire forecasting models [21], an evacuation guiding and an updated building map. With all this data he is able to give commands to his crew operating at the incident. Furthermore, the sensor data is used to update the building model and to generate a detailed intervention file, as discussed before.
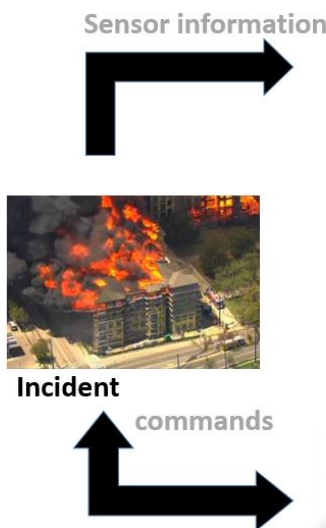

Sensor informatio

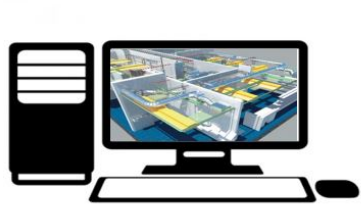

BIM module

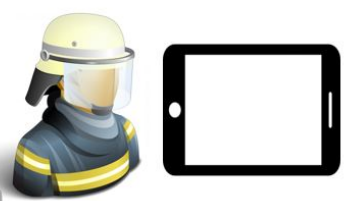

Commander
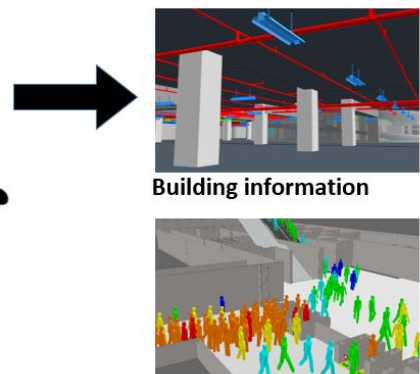

Evacuation guiding

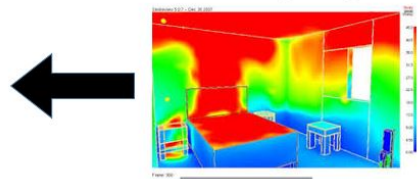

Fire forecasting

Fig. 2. Future use of BIM during incident management: sensor visualization, fire forecasting management, evacuation planning and building information visualization. 


\section{LOW-COST VIDEO SENSING FOR SITUATIONAL AWARENESS AND BIM OPTIMIZATION}

Although there is a lot of information available through the BIM models it is import to remark that there could arise several problems when only focusing on BIM, e.g., the model could be outdated or inaccurate. Furthermore, the actual state of the fire is also an important factor in analyzing and generating an overview of the fireground. Based on these issues, you can not completely rely on building information models and additional real-time sensing data is needed to perform situational awareness. Endsley et al. [22] defined situational awareness as the perception of the elements in the environment within a volume of time and space, the comprehension of their meaning and the projection of their status in the near future. The perception of the environment is what we tackle within this paper using computer vision techniques, as addressed in Section 5.

Recently, there appear more and more embedded or smart phone attachable sensors that are useful for situational awareness. The cost of these devices is decreasing and the amount of consumer applications is increasing. The majority of these systems, however, are still in an exploratory phase and are mostly not commercially available. There are some research tracks that explore the possibilities of these devices in case of a fire for real-time sensing, and the road map of NIST [23] gives some examples for further research on this topic. One example is the Moses (Mobile sensing for fire safety) research project ${ }^{3}$. This project focuses on real-time situation awareness for fire fighters. The localization is estimated with foot mounted inertial sensors and a GPS sensors. This is further mapped on a 2D map of the building. The disadvantage of this approach once again is that the map needs to be up-to-date and that there is no feedback of the actual state of the building. Furthermore, the Moses project uses probability calculations for the evaluation of personal and health sensing information such as the heart rate and the breathing speed. Similarly, the heat stress risk could be calculated with wearable sensors and the core temperature [24]. A last interesting aspect of the Moses project is the tactical visualization of the data on a tablet. The commander in chief gets and overview of the location of the firefighters on a 2D map. Future research could incorporate actual visibility estimations [25] in the platform.

Another example of the use of real-time sensing is the work of Feese et al. [26]. They used smartphone data to monitor firefighter performance for post-fire analysis. The project focused on the monitoring of firefighters health during missions, monitoring of the environment of firefighters for toxic gases and high temperatures, and providing navigational support. Furthermore, The SmartRescue app from Lazreg et al. [27] used smartphone sensors along with a Bayesian network classifier to assess the fire situation and to predict the further development, as well as to support an indoor positioning system. Within their application, the indoor localization is estimated by the strength of the WIFI signal. The limitation of this approach is that the system heavily relies on the WIFI signal which could be unavailable in case of a fire. In Section 5 we will give some solutions to this problem. Also in the work of Li et al. [28] there are several systems evaluated for indoor positioning. Finally, it is important to remark that the usability and the interaction of the applications need to be evaluated. Parvaneh et al. [1] performed a study on this for the GDACS mobile and the SmartRescue app and give some valuable practical rules which we will also integrate to optimize our frameworks' user experience.

Besides the research on the application of low-cost smartphone sensors in a firefighting context there are some research tracks on additional wearable sensors. Globes WASP ${ }^{4}$ (wearable advanced sensor platform) developed a commercial device that consist of a t-shirt for monitoring bio-metrics and a belt for location tracking. The Smart@Fire project ${ }^{5}$ on the other hand focuses on the creation of smart personal protective systems. Although all the possibilities with the wearable and smartphone sensors, however, no fire crew actively uses these systems. This is mostly due to the lack of reliability and the complexity of the systems to handle. A system which is well established is the thermal imaging device and there is research going on to make the handling of the thermal devices easier. Scott Sight built an in-mask thermal sensor ${ }^{6}$ and Vizir ${ }^{7}$

\footnotetext{
${ }^{3}$ http://www.techforfuture.nl/53-MoSeS_Mobile_Sensing_for_Safety.html

${ }^{4}$ http://globeturnoutgear.com/innovation/wasp

${ }^{5} \mathrm{http}: / / \mathrm{www}$. smartatfire.eu/upcoming-events.aspx

${ }^{6} \mathrm{http}: / / \mathrm{www} . \mathrm{scottsafetynation.com/scott-sight-nfpa-1981-certified-now-shipping/}$

${ }^{7}$ http://www.darix.ch/
} 
offers a hands-free operation mask. There are some differences between both systems, i.e., the Scott Sight has no external camera and a thermal viewer in the corner, while the VIZIR has its augmented reality images in the center. Further research will be necessary to achieve full fireground understanding from these devices. Currently, there is no interaction with different crews and the information seen on the screen is not automatically interpreted. To handle this issue, some recent papers try to make the thermal imaging more interactive. Paugam et al. [29], for example, use the hand held thermal devices to derive fire radiative power (FRP) and flame front rate of spread (ROS) in case of a wildland fire. Kim et al. [30] use a probabilistic classification to classify fire and smoke regions from a single thermal camera. These results are used to autonomously navigate a firefighting robot towards the fire and are evaluated in a realistic fire set-up. In our framework we will use the smoke and fire classification of a thermal imager for accurate fireground understanding.

\section{LOCATION ESTIMATION AND UNDERSTANDING}

In addition to the active monitoring of the fire fighters' performance it is important to get a clear view on their environment. Although the active use of thermal devices, however, there is currently no system available to perform automated fire location understanding. In this section we describe some technological innovations that could help to create an automated overview of the environment during intervention. Each of these technologies will contribute in transforming the firefighting into a more sensing and data-driven discipline.

Indoor localization and location understanding are very hot topics. There is a tendency in the visual domain to create more and more detailed 3D models, although their generation is computational expensive. Furthermore, in case of a fire, the visual view is reduced or unavailable due to the smoke particles. This will result in a failure of standard SLAM (simultaneous location and mapping) algorithms [31]. Although, these limitations are widely recognized, there is done already some research in indoor location estimation during a fire. An overview of these systems that can be used in emergency situations is described by Bastos et al. [32] and Starr et al. [33]. Furthermore, Emilsson et al. [31] used a stereo-pair of thermal images with IMU (inertial measurement unit) information to create an overview map or a 3D model of visited environments. From our point of view, however, it is not necessary to create a detailed 3D or complex point cloud model for fire location understanding. In our framework, contrarily, we focus on computer vision based object detection and localization and semantically compare these results with the location of objects in the building model. Based on their semantic similarity, an estimation of the indoor location is given.

As illustrated in Figure 3, our object detection module is also helpful for understanding what is burning and where it is located. The input of the system could be a simple monocular view of a hand-held thermal camera. The burning object can then be linked to a map of the location and surrounding object annotations will facilitate the interpretation of the fire. Finally, the output of our system could be (for instance) the following text message: "The fridge is burning in the left corner of the cellar. The fire is located in the bottom right of the fridge and there is a hot smoke layer of $1 \mathrm{~m}$." To achieve this final goal, i.e., automatically generating text messages of the fire status, we focus on the following building blocks:

- An object detection and recognition module;

- A scene classification block for location estimation verification;

- $\quad$ A room layout estimation block;

- $\quad$ A smoke-fire location estimation block.

In the next subsections, an overview is given of the selected state-of-the art mechanisms for each of these building blocks. Important to remark is that our current evaluation is done on visual images. However, future work will apply the similar techniques on thermal images and evaluate its accuracy within this context. Of course, only objects which irradiate infrared light will be able to be detected and localized. 
The work of Lu et al. [12] showed the possibility of transfer learning from visual and thermal images. Transfer learning is a general technique in machine learning where a pretrained system for a particular task or domain (e.g. classification of birds) is fine-tuned on another task (e.g. classification of dogs). The main advantage of this approach is that it achieves high accuracy for the new task with a limited set of labeled training examples [34]. Finally it is important to remark that the evaluation and optimization of each of the following building blocks will be done separately based on their precision, recall and accuracy.

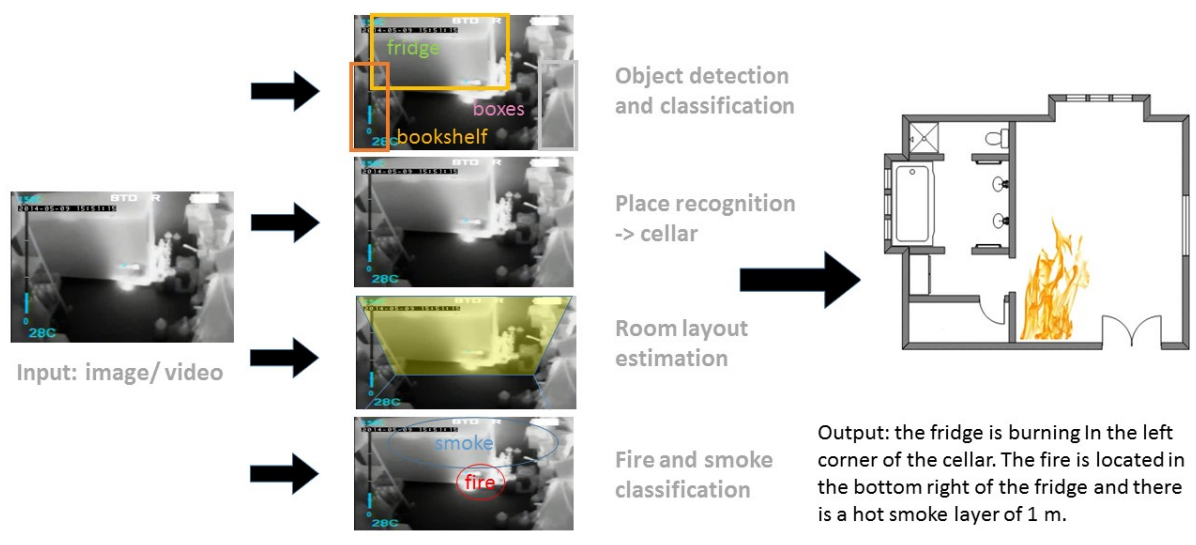

Fig. 3. Thermal image analysis for fireground understanding.

\subsection{Object recognition and detection for location estimation}

The first building block that we want to address is the object recognition and detection module. Object detection could be described as 'where is the object located in the image', whereas object recognition identifies to which semantic class an object belongs. Localization of objects in a real fire could be helpful in two ways. Firstly, an automated detection and reporting of the object that is burning could help to get a faster overview of the scene. The information of the specific object that is burning could also be used to deduct the heat release rate in a fire forecasting framework. Secondly, objects are important for localization and location understanding in reduced visibility environments. The idea of combining building information with object recognition is already addressed in literature. Kropp et al. [35] have used this to identify the progress of the building process and Apollonio et al. [36] address the possibilities of object detection in raw-data to link with existing building models.

The most difficult problem for object detection is that there are a lot of variances in object appearance due to illumination, viewpoint, and non-rigid deformation. Furthermore, in the thermal domain the amount of variances is increasingly higher. In the visual domain it is a popular approach to use multi-labeling convolutional neural network architectures to cope with these issues. Currently faster-RCNN [37] is used in our set-up to identify objects and their location. To get similar results in the thermal domain, we will need to apply a transfer learning strategy, like proposed by Lu et al.[12]. The faster-RCNN network consist of two modules, firstly a deep fully convolutional network for region proposals and secondly a fast R-CNN detector for end-to-end training. The last block will classify the region proposals into object categories or as background. In our context, the network is trained and evaluated on the Microsoft COCO visual object detection dataset [38]. Irrelevant object classes, such as tie, train, and bird are removed. Future work will investigate the impact of using the recently released ADE20K dataset ${ }^{8}$ to train the object recognition especially on scene contexts

\footnotetext{
${ }^{8}$ http://groups.csail.mit.edu/vision/datasets/ADE20K/
} 
and evaluate object detection in the thermal domain. Finally, some example outputs of the object detection module on the COCO dataset are given in Figure 4. These results also indicates some difficulties, i.e., not all the objects in the image are detected and some objects are incorrect classified (e.g., sofa should be bed).
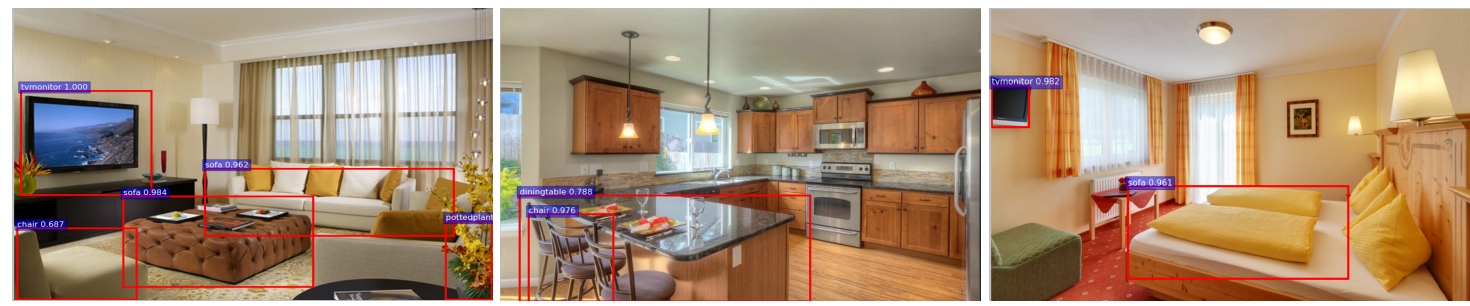

Fig. 4. Object detection with faster-RCNN module [37] from online room images

\subsection{Scene classification}

Being able to classify the scene type in the field of view of the camera will facilitate the location estimation and understanding. Due to differences in object types, shapes and texture features that construct the scene, this is sitll a challenging task. Recent deep learning based approaches, however, seem to be able to perform the classification with high accuracy. By embedding this semantic information of the scene in the room layout estimation, better estimations can be made in the localization process. As such, when the scene type can be detected, it is included in this process.

Over the last decade, several literature studies have already shown impressive results regarding the classification of indoor and outdoor scenes [39], and more recent studies have added the ability to predict more detailed attributes, such as the scene type, e.g., kitchen, living room, or bed room. Zhou et al.[40], for example, constructed a new scene-centric database named Places, containing over 7 million labeled pictures of scenes distributed over 365 classes. Currently, the state-of-the-art systems for scene classification are based on CNN (convolutional neural networks) trained networks, mostly trained using a transfer learning approach on the Imagenet models. The current highest top-5 accuracy achieved on this Places dataset is 0.8491 , using the VGG deep convolutional neural network [41]. Figure 5 shows the output of this scene classification approach on our test images. Similar to the object detection module we get some missclassification due to the strong correlations between two (sub-)classes, e.g., hotel room and bedroom. These errors can possibly be removed by incorporating spatio-temporal BIM-based history of the localization on the floorplan.

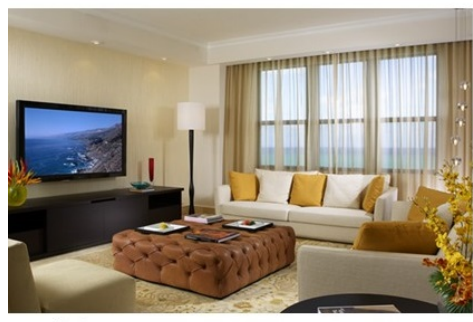

living_room: 0,58 , parlor: 0,40

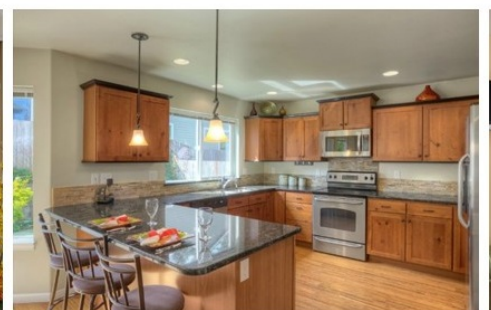

kitchen: 0,60 ,kitchenette: 0,39

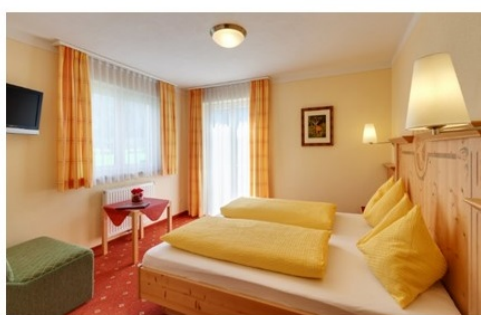

hotel_room: 0,85 , bedroom: 0,10

Fig. 5. Scene classification with a VGG network from online room images [41] 


\subsection{Room layout estimation for location verification}

In thermal domain, objects are hard to detect due to their lower resolution and limited 'texture' features, i.e., objects share similar temperature values which leads to higher similarity accross objects or even worser, object merging. In order to cope with these issues, a thermal domain room layout estimation can be performed. This module focuses on estimating the 3D cuboid that defines the room, i.e., inferring the location and position of the walls and the ceiling. Based on this cuboid, an estimation of the location and position of the sensor could still be made. Existing solutions for layout estimation mostly rely on hand-crafted features and vanishing lines and they often fail in highly cluttered rooms. More interesting are the approaches of Ren et al. [42] and Delay et al. [43]. Ren et al., on the one hand, propose a coarse-to-fine strategy for indoor layout estimation. For a monocular input indoor image, a coarse layout estimation is generated with a multi-task fully convolutional neural network (MFCN). An example of this technique is shown in Figure 6, where the blue-green lines corresponds to the coarse layout estimation. In the second step, occluded lines and missing lines are filled and possible layout choices are ranked according to a predefined score function in the second stage. Delay et al., on the other hand, use a fully convolutional neural network (FCNN) in conjunction with a novel optimization framework for generating layout estimations. Further evaluation and adaption of both techniques will be necessary to accurately apply the techniques of Ren and Delay et al. on thermal images.
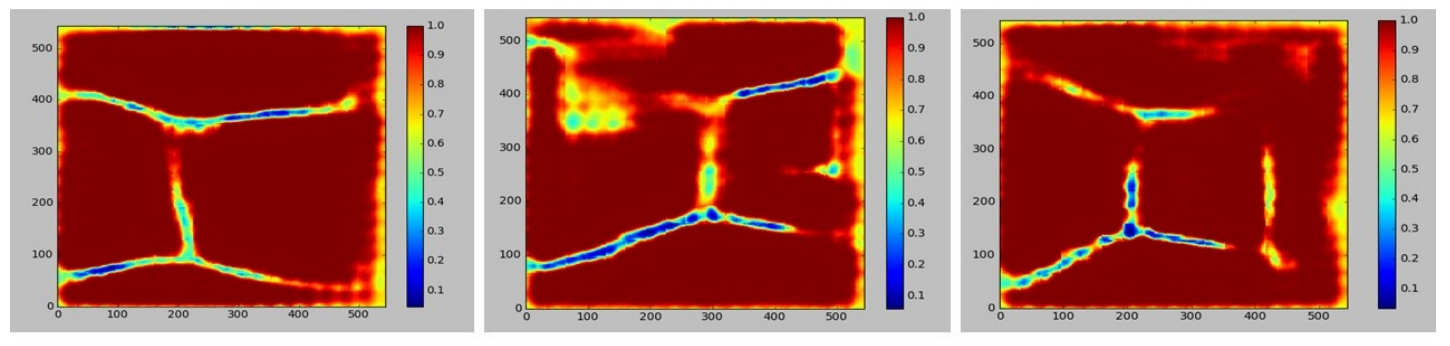

Fig. 6. Thermal imaging output of the coarse layout estimation with a fully convolutional neural network.

\subsection{Smoke and fire classification}

The last module of the architecture proposed in Figure 3 performs smoke and fire classification. In Kim et al. [30], for example, the output of such a classifier is used for autonomous firefighting robot guiding. By using Bayesian probabilistic classification on low-level image features a decent differentiating between smoke, fire and thermal reflections in infrared images could be made. A general overview of state-of-the-art mechanisms for fire detection in the visual, infrared and multimodal spectrum is given in Cetin et al. [44]. The majority of these methods are feature engineering based approaches. In future work, we also want to explore the possibilities of deep learning architectures for this classification task. Those techniques have already proven to give decent results in general classification and segmentation tasks [45]. Furthermore, the possibilities of combining thermal and visual images for the understanding and fire localization [46] task will be further explored. Besides the detection their are also measurements possible to link the actual fire size or smoke height to fire forecasting modules [21]. 


\section{Semantic linking of video annotations with building information}

The previous sections have given an overview of several mechanisms to generate semantic information from visual and thermal images. Within this section we describe how these semantic annotations from the scene classification, object detection and room layout estimation can be linked/matched to the semantic descriptions of the building models.

In the current set-up, the existing sample project of the Revit package is used as our information model and the data is transformed into a textual ifcXML file. Firstly, the scene labels are extracted from the image (parlor: 0.44, living: 0.17, lobby: 0.10, waiting room: 0.06) and for each of the labels a weighted search is performed on the BIM-based XML-file and, taking into account the spatio-temporal history of the localization, the room with the highest matching score is selected. Secondly, the objects are detected in the image (chair, sofa, bottle) and with these objects we perform a confirmation of the previous step and roughly estimate the position of the imaging sensor in the room. Thirdly, this positioning is further refined using our coarse room layout estimation and a final (improved) estimate of the viewpoint is generated (as indicated by the red arrow). Finally, the location of the camera is timestamped on the building model and the spatio-temporal filter in step 1 is updated. If available, additional sensors such as an inertial measurement unit (IMU) can also be used to verify the retrieved location. Furthermore, it is important to remark that the building model can be updated with the detected objects in case they are not incorporated in the current version of the model.

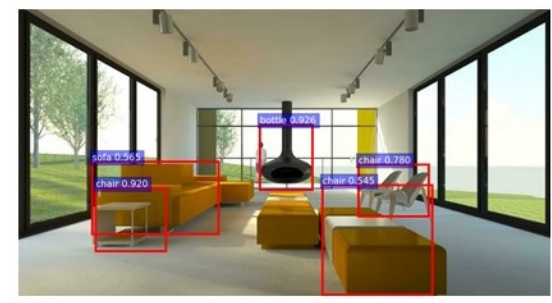

Scene object detection

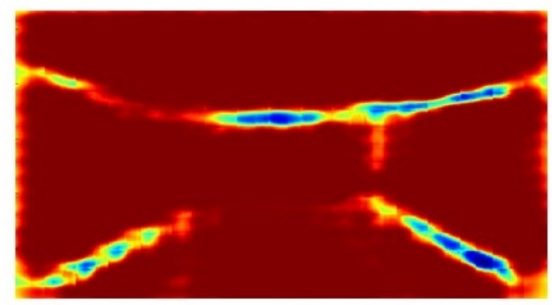

Room layout estimation

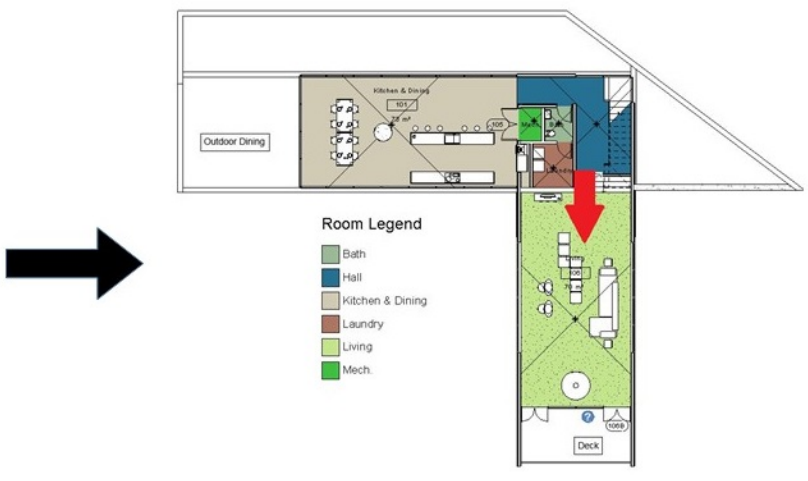

Location retrieval in building model

Fig. 7. Scene classification, object detection and room layout estimation for location retrieval (red arrow) in a building model

Once the location in the building model is known it is possible to update the model with fire-related sensor data (such as the visibility level and the smoke height) for visualization or for evacuation purposes. Furthermore, there could be an indication of the object that is burning and the fire properties of that object could be derived from the building model. Future work will focus on the use of this information for fire forecasting. Finally, it is important to remark that we are currently collecting several building models and geotagged video footages to evaluate the proposed localization mechanism in both the visual and thermal domain. The last step will be the collection of video footages within real-fire experiments to facilitate fire incident management. 


\section{CONCLUSION}

In this paper we proposed an outline for the combination of state-of-the-art computer vision techniques and building information models (BIM) for facilitating the localization and situational awareness problem in fire emergency situations. The novelty of this paper is the semantic matching of visual object recognition results from images with data management tools for building information models. To the best of our knowledge, this is the first literature review that is describing building models for fire safety and incident management. Important to remark is that the proposed framework is still work in progress and many aspects (especially for thermal image analysis) need to be further explored and evaluated before being applicable in a real firefighting set-up. However, this paper shows the technology readiness of the majority of the proposed building blocks and is a starting point to make the firefighting more sensing and data-driven.

\section{FUTURE WORK}

Future research will investigate the possibilities of transfer learning for applying visual domain techniques, as proposed in this paper, on thermal domain images. However, more datasets and thermal imaging object models will be needed to evaluate the proposed system and optimize each of its building blocks. The dataset of Lu et al. [12] with visual and thermal images of indoor scenes will be used to train and validate our proposed mechanism in combination with geotagged videofootages and building models. Furthermore, videofootages of real-fire experiments are certainly useful to validate our proposed framework. In addition, handheld thermal devices introduce motion artefacts and need to cope with fast changing views, which probably will also have an impact on the detection results. More research will be done in this field. Furthermore, object detection will become harder due to the lower resolution of these kind of imagers. Finally, it is imporant to remark that building models need to be up to date, complete and rich in detail. Currently, however, the building models are only used during construction and are mostly not updated afterwards, limiting their practical applicability. However, we expect that this will change in the upcoming years since more and more researchers start to explore the link with BIM in a wide range of application domains.

\section{ACKNOWLEDGMENTS}

The research activities as described in this paper were funded by Ghent University through GOA project BOF16/GOA/004.

\section{REFERENCES}

[1] Parvaneh Sarshar, Vimala Nunavath, and Jaziar Radianti. On the usability of smartphone apps in emergencies. In International Conference on Human-Computer Interaction, pages 765-774. Springer, 2015.

[2] Vimala Nunavath and Andreas Prinz. Taking the advantage of smartphone apps for understanding information needs of emergency response teams for situational awareness: Evidence from an indoor fire game. In International Conference on Human-Computer Interaction, pages 563-571. Springer, 2016.

[3] Florian Vandecasteele, Bart Merci, and Steven Verstockt. Reasoning on multi-sensor geographic smoke spread data for fire development and risk analysis. Fire Safety Journal, (86):65-74, 2016.

[4] Chuck Eastman, Charles M Eastman, Paul Teicholz, Rafael Sacks, and Kathleen Liston. BIM handbook: A guide to building information modeling for owners, managers, designers, engineers and contractors. John Wiley \& Sons, 2011. 
Yang Zou, Arto Kiviniemi, and Stephen W Jones. A review of risk management through bim and bim-related technologies. Safety Science, 2016.

[6] Chris Jelenewicz et al. Building information modeling and fire protection. In ASSE Professional Development Conference and Exposition. American Society of Safety Engineers, 2013.

[7] Shih-Hsu Wang, Wei-Chih Wang, Kun-Chi Wang, and Shih-Yu Shih. Applying building information modeling to support fire safety management. Automation in Construction, 59:158-167, 2015.

[8] Peng Zhou and Nora El-Gohary. Ontology-based automated information extraction from building energy conservation codes. Automation in Construction, 74:103-117, 2017.

[9] Chengde Wu, Saied Zarrinmehr, Mohammad Rahmani Asl, and Mark J Clayton. Facilitating fire and smoke simulation using building information modeling. In International Conference on Computer-Aided Architectural Design Futures, pages 366-382. Springer, 2015.

[10] Lee-Kuo Lin, Yu-Huai Hsu, and Yu-Chen Hsiao. A study of applying bim technique into fire disaster investigation system. In 2016 Eighth International Conference on Measuring Technology and Mechatronics Automation (ICMTMA), pages 32-35. IEEE, 2016.

[11] Steffen Franz and Uwe Rüppel. Supporting forensic fire cause analysis with next generation mobile devices by assembling a bim based on multiple integrated sensor data. In 22nd EG-ICE International Workshop, July 2015.

[12] Guoyu Lu, Yan Yan, Li Ren, Philip Saponaro, Nicu Sebe, and Chandra Kambhamettu. Where am $\mathrm{i}$ in the dark: Exploring active transfer learning on the use of indoor localization based on thermal imaging. Neurocomputing, 173:83-92, 2016.

[13] Rosalie Faith Wills. Development of a Cyber Physical System for Fire Safety. PhD thesis, 2015.

[14] MJ Spearpoint. Fire engineering properties in the ifc building product model and mapping to branzfire. 2006.

[15] Albert Y Chen and James C Chu. Tdvrp and bim integrated approach for in-building emergency rescue routing. Journal of Computing in Civil Engineering, page C4015003, 2015.

[16] Sisi Zlatanova, George Vosselman, Kourosh Koshelham, Rob Peters, Bart Beers, Robert Voûte, Bart De Lathouwer, Matty Lakerveld, H Djurrema, and G Spaling. Slimme 3d indoormodellen ter ondersteuning van crisismanagement in grote openbare gebouwen. 2015.

[17] Tee-Ann Teo and Kuan-Hsun Cho. Bim-oriented indoor network model for indoor and outdoor combined route planning. Advanced Engineering Informatics, 30(3):268-282, 2016.

[18] Hongxia Wang, A Gluhak, and H Tafazolli. Integration of bim and live sensing information to monitor building energy performance. In CIB W78 International Conference, Beijing, 2013.

[19] Jianli Chen, Tanyel Bulbul, John E Taylor, and Guney Olgun. A case study of embedding realtime infrastructure sensor data to bim. In Construction Research Congress 2014: Construction in a Global Network, pages 269-278, 2014.

[20] Muhammad Arslan, Zainab Riaz, Adnan Khalid Kiani, and Salman Azhar. Real-time environmental monitoring, visualization and notification system for construction h\&s management. Journal of Information Technology in Construction (ITcon), 19(4):72-91, 2014.

[21] Tarek Beji, Steven Verstockt, Rik Van de Walle, and Bart Merci. On the use of real-time video to forecast fire growth in enclosures. Fire Technology, 50(4):1021-1040, 2014.

[22] Mica R Endsley. Measurement of situation awareness in dynamic systems. Human Factors: The Journal of the Human Factors and Ergonomics Society, 37(1):65-84, 1995.

[23] Hernandez Paul. Research roadmap traces the path to'smart'fire fighting. 2015. 
[24] Mitra Baratchi, Lennart Teunissen, Peter Ebben, Wouter Teeuw, Jan Laarhuis, and Maarten van Steen. Towards decisive garments for heat stress risk detection. In Proceedings of the 2016 ACM International Joint Conference on Pervasive and Ubiquitous Computing: Adjunct, pages 10951100. ACM, 2016.

[25] Florian vandecasteele, Steven Verstockt, and Bart Merci. Smoke behaviour analysis with multiview smoke spread data. In Interflam 2016: fire science and engineering conference, volume 1, pages 399-408. interscience communications, 2016.

[26] Sebastian Feese, Bert Arnrich, Gerhard Troster, Michael Burtscher, Bertolt Meyer, and Klaus Jonas. Coenofire: monitoring performance indicators of firefighters in real-world missions using smartphones. In Proceedings of the 2013 ACM international joint conference on Pervasive and ubiquitous computing, pages 83-92. ACM, 2013.

[27] Mehdi Ben Lazreg, Jaziar Radianti, and Ole-Christoffer Granmo. Smartrescue: Architecture for fire crisis assessment and prediction.

[28] Nan Li, Burcin Becerik-Gerber, Lucio Soibelman, and Bhaskar Krishnamachari. Comparative assessment of an indoor localization framework for building emergency response. Automation in Construction, 57:42-54, 2015.

[29] Ronan Paugam, Martin J Wooster, and Gareth Roberts. Use of handheld thermal imager data for airborne mapping of fire radiative power and energy and flame front rate of spread. IEEE Transactions on Geoscience and Remote Sensing, 51(6):3385-3399, 2013.

[30] Jong-Hwan Kim and Brian Y Lattimer. Real-time probabilistic classification of fire and smoke using thermal imagery for intelligent firefighting robot. Fire Safety Journal, 72:40-49, 2015.

[31] Erika Emilsson and Joakim Rydell. Chameleon on firethermal infrared indoor positioning. In 2014 IEEE/ION Position, Location and Navigation Symposium-PLANS 2014, 2014.

[32] Arivan S Bastos, Vaninha Vieira, and Antonio L Apolinário Jr. Indoor location systems in emergency scenarios-a survey. In Proceedings of the annual conference on Brazilian Symposium on Information Systems: Information Systems: A Computer Socio-Technical Perspective-Volume 1, page 34. Brazilian Computer Society, 2015.

[33] Joseph W Starr and BY Lattimer. Evaluation of navigation sensors in fire smoke environments. Fire Technology, 50(6):1459-1481, 2014.

[34] Brian Chu, Vashisht Madhavan, Oscar Beijbom, Judy Hoffman, and Trevor Darrell. Best practices for fine-tuning visual classifiers to new domains. In Computer Vision-ECCV 2016 Workshops, pages 435-442. Springer, 2016.

[35] Christopher Kropp, Markus König, and Christian Koch. Object recognition in bim registered videos for indoor progress monitoring.

[36] Fabrizio Ivan Apollonio, Marco Gaiani, and Zheng Sun. A reality integrated bim for architectural heritage conservation. Handbook of Research on Emerging Technologies for Architectural and Archaeological Heritage, page 31, 2016.

[37] Ross Girshick. Fast r-cnn. In Proceedings of the IEEE International Conference on Computer Vision, pages 1440-1448, 2015.

[38] Tsung-Yi Lin, Michael Maire, Serge Belongie, James Hays, Pietro Perona, Deva Ramanan, Piotr Dollár, and C Lawrence Zitnick. Microsoft coco: Common objects in context. In European Conference on Computer Vision, pages 740-755. Springer, 2014.

[39] Jiebo Luo and Andreas Savakis. Indoor vs outdoor classification of consumer photographs using low-level and semantic features. In International Conference on Image Processing (ICIP), volume 2, pages 745-748, 2001. 
[40] Bolei Zhou, Agata Lapedriza, Jianxiong Xiao, Antonio Torralba, and Aude Oliva. Learning deep features for scene recognition using places database. In Advances in Neural Information Processing Systems (NIPS), pages 487-495, 2014.

[41] Limin Wang, Sheng Guo, Weilin Huang, and Yu Qiao. Places205-VGGNet models for scene recognition. arXiv:1508.01667, 2015.

[42] Yuzhuo Ren, Chen Chen, Shangwen Li, and C-C Jay Kuo. A coarse-to-fine indoor layout estimation (cfile) method. arXiv preprint arXiv:1607.00598, 2016.

[43] Saumitro Dasgupta, Kuan Fang, Kevin Chen, and Silvio Savarese. Delay: Robust spatial layout estimation for cluttered indoor scenes. In Proceedings of the IEEE Conference on Computer Vision and Pattern Recognition, pages 616-624, 2016.

[44] A Enis Cetin, Bart Merci, Osman Günay, Behçet Ugur Töreyin, and Steven Verstockt. Methods and Techniques for Fire Detection: Signal, Image and Video Processing Perspectives. Academic Press, 2016.

[45] Karen Simonyan and Andrew Zisserman. Very deep convolutional networks for large-scale image recognition. arXiv preprint arXiv:1409.1556, 2014.

[46] Steven Verstockt, Alexander Vanoosthuyse, Sofie Van Hoecke, Peter Lambert, and Rik Van de Walle. Multi-sensor fire detection by fusing visual and non-visual flame features. In International Conference on Image and Signal Processing, pages 333-341. Springer, 2010. 